\title{
MURCIÉLAGOS (CHIROPTERA) EN ÁREAS VERDES URBANAS DE LA CIUDAD DE TEPIC, NAYARIT, MÉXICO
}

BATS (CHIROPTERA) IN URBAN GREEN SPACES FROM TEPIC CITY, NAYARIT, MEXICO

\author{
Juan Pablo Ramírez-Silva ${ }^{1}$ | Elsa Jazmín Lugo-GiL²
}

\begin{abstract}
${ }^{1}$ Maestría en Ciencias para el Desarrollo, Sustentabilidad y Turismo. Unidad Académica de Turismo. Universidad Autónoma de Nayarit. Ciudad de la Cultura "Amado Nervo”. Tepic, Nayarit. México. C.P. 63155.
\end{abstract}

\begin{abstract}
${ }^{2}$ Unidad Académica de Agricultura. Km 9 carretera Tepic-Compostela, Xalisco, Nayarit. México. C.P. 63155.
\end{abstract}

\section{ResUmen}

Los murciélagos desempeñan un papel fundamental en los ecosistemas, ya que efectúan múltiples interacciones bióticas como la dispersión de semillas, polinización y el control de plagas, además de que poseen hábitos alimenticios muy variados. En Nayarit se han registrado 67 especies de quirópteros, pero ningún estudio se ha enfocado en las zonas urbanas del estado. Nuestro objetivo fue evaluar la diversidad y abundancia de murciélagos en las áreas verdes de la zona urbana de Tepic, Nayarit. Se realizó un muestro en nueve sitios por medio de una red de 12 metros, colocada de 18:00 h a 23:00 h., durante el periodo de noviembre del 2015 a noviembre del 2016, con un total de 45 horas/red. Se muestrearon tres tipos de hábitat urbanos: plazas, zanjones y parques. Con los datos recabados se estimó el índice de diversidad alfa de Shannon y se realizó una prueba de t modificada por Hutchenson para comparar

Revisado: 07 de mayo de 2020; aceptado: 10 de junio de 2020; publicado: 15 de julio de 2020. Autor de correspondencia: Juan Pablo Ramírez Silva, pablor@uan.edu.mx

Cita: Ramírez-Silva, J.P. y E.J. Lugo-Gil. 2020. Murciélagos (Chiroptera) en áreas verdes urbanas de la ciudad de Tepic, Nayarit, México. Revista Mexicana de Mastozoología, nueva época, 10(1):21-31. ISSN: 2007-4484.www.revmexmastozoologia.unam.mx

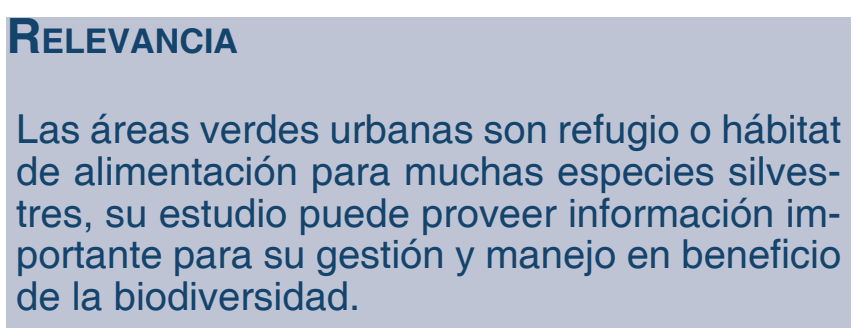

los distintos ecosistemas. Se registraron tres especies de murciélagos (Artibeus lituratus, Artibeus jamaicensis y Artibeus hirsutus). Los parques fueron el ecosistema que registró la mayor diversidad y abundancia de murciélagos, mientras que la especie que se documentó con mayor frecuencia fue A. lituratus.

Palabras clave: ambientes antropizados, parques urbanos, quirópteros.

\section{Abstract}

The bats play a fundamental role in the ecological processes of ecosystems, they carry out multiple biotic interactions such as seed dispersion, pollination, and pest control, they also have very varied feeding habits. In the Mexican state of Nayarit have been registered 67 bat species, but no one research has focused on the urban areas. Our objective was to research the species present in the green areas of the city of Tepic, Nayarit. Nine sites were sampled using a 12-meter 
network, which was placed from 18:00 h to 23:00. The samplings were carried out from November 2015 to November 2016, for a total of 45 hours/ network. Three types of habitat were covered: squares, zanjon, and parks. We performed the Shannon alpha index to assess diversity and a t-test was used to compare between the different habitats. Three species were registered (Artibeus lituratus, Artibeus jamaicensis and Artibeus hirsutus). The urban ecosystem with the greatest diversity and abundance was the parks and the species that presented the highest frequency in the urban area was $A$. lituratus.

Key words: anthropized areas, bats, green spaces.

\section{INTRODUCCIÓN}

Los murciélagos son un grupo de mamíferos pertenecientes al orden Chiroptera, el cual cuenta con más de 1,300 especies clasificadas en 17 Familias que incorpora aproximadamente al $20 \%$ de todas las especies de mamíferos del mundo. Es considerado el segundo grupo con mayor número de especies, superado solamente por el orden Rodentia (Álvarez-Castañeda et al., 2015). Los murciélagos desempeñan un papel fundamental en los ecosistemas, debido a que sus variados hábitos alimenticios forman parte importante de los procesos ecológicos regionales. Llevan a cabo múltiples interacciones bióticas que contribuyen al control de plagas, la dispersión de semillas y polinización, entre otros (Greenhall, 1982).

La destrucción del hábitat es una de las principales causas por las cuales los quirópteros se encuentran amenazados (Díaz et al., 2013), particularmente la urbanización ha provocado cambios drásticos en la vegetación, temperatura ambiental, hidrología y otros factores que afectan la dinámica poblacional de las especies animales; en estas condiciones los recursos como el alimento y hábitat son reducidos (Sierra, 2012). A pesar del impacto negativo que generan las zonas urbanas sobre la diversidad biológica, existen espacios como las áreas verdes, que usualmente son empleados como zona de recreación para los humanos y que desempeñan una función muy importante como refugio para algunas especies de fauna silvestre (Arias-Aguilar, 2015). No obstante, para que un área verde pueda contribuir efectivamente con la conservación de la flora y fauna, requiere cumplir ciertas características, entre las que destacan el tipo y estructura de la vegetación, así como su ubicación y tamaño. Se ha observado que las áreas de mayor dimensión, con vegetación nativa y con poca perturbación son capaces de alojar una mayor riqueza de especies de fauna silvestre (García-Méndez et al., 2014).

Asimismo, las áreas verdes podrían tener una función determinante en la manutención de las poblaciones de murciélagos, ya que algunos estudios han documentado que las áreas suburbanas proporcionan hábitat de alimentación para las poblaciones de murciélagos (Rhodes y Catterall, 2008; Threlfall et al., 2012). Normalmente, la cubierta de suelo urbano disminuye considerablemente la actividad de estos organismos, así como la riqueza de especies (Gonsalves et al., 2013; Hourigan et al., 2006; Luck et al., 2013).

Las publicaciones sobre murciélagos en entornos urbanos son escasas. La mayor parte de ellos han sido realizados en localidades de Europa y América del Norte, en los cuales se abordan aspectos sobre la ecología de los murciélagos en relación con distintos tipos de infraestructura tales como, los edificios antiguos, el alumbrado público, las zonas industriales y algunas plazas. En contraparte, en las zonas neotropicales los estudios de murciélagos en áreas urbanas se enfocan principalmente a la descripción de la riqueza y abundancia de especies (Jung y Threlfall, 2016).

Tepic es una ciudad relativamente pequeña en relación con otras ciudades en México, de hecho, es una de las 12 capitales menos pobladas del país, con una población de 332,863 habitantes (INEGI, 2010). Se sitúa en un valle rodeado por cerros con vegetación que en su mayoría es nativa y que en general presenta buenas condiciones de conservación. Todavía es común observar especies silvestres en distintas zonas de la ciudad, lo cual hace suponer que murciélagos podrían estar haciendo uso de las áreas verdes. Sin embargo, hasta el momento se cuenta con muy poca información al respecto; por ejemplo, ¿cuáles son las especies de quirópteros que habitan en la ciudad? ¿se encuentran las mismas especies en los distintos tipos de áreas verdes? o ¿en dónde se localiza la mayor diversidad de murciélagos? En este estudio se realizó un 
muestreo preliminar para resolver estas interrogantes y sentar la base para estudios de conservación de la biodiversidad urbana.

\section{Método}

\section{Área de estudio}

El estado de Nayarit posee una superficie de $27,857 \mathrm{~km}^{2}$, que se localiza en la porción noroeste de México. Colinda al norte con los estados de Sinaloa y Durango, al este con Durango, Zacatecas y Jalisco, al sur con Jalisco y el océano Pacífico y al oeste con el océano Pacífico y Sinaloa (INEGI, 2017). En la mayor parte del estado se presenta un clima cálido subhúmedo, solo en el $6 \%$ del territorio se presenta un clima templado subhúmedo que corresponde a las sierras, el $2 \%$ es seco y semiseco con una ubicación hacia el sur y sureste del estado y un $0.5 \%$ es cálido húmedo. Registra una temperatura anual media de $25^{\circ} \mathrm{C}$; la temperatura mínima es de $12^{\circ} \mathrm{C}$ en enero y la máxima es de $35^{\circ} \mathrm{C}$ durante los meses de mayo y junio. Presenta lluvias durante el verano en los meses de mayo a septiembre con una precipitación media de 1,100 mm anuales.

El municipio de Tepic es la capital del estado, el cual se encuentra entre los paralelos $21^{\circ} 23^{\prime}$ y $21^{\circ} 52^{\prime}$ de latitud norte, los meridianos $104^{\circ} 35^{\prime}$ y $105^{\circ} 09^{\prime}$ de longitud oeste. Cuenta con una altitud entre 400 y 2,300 msnm y ocupa el $5.96 \%$ de la superficie del estado. La ciudad de Tepic tiene una superficie de $1657 \mathrm{~km}^{2}$, se encuentra en la zona centro sur del municipio, colindando con la localidad de Camichín de Jauja, municipio de Tepic y con el municipio de Xalisco, Nayarit.

El trabajo de campo se realizó en tres tipos de ecosistemas urbanos: plazas, zanjones y parques (Figura 1A, 1B y 1C), cuyas características se definen a continuación:

\section{Plazas}

Son definidas como áreas abiertas al público, con construcciones en uso como jardineras rodeadas por edificios, presentan áreas en bloque cubiertos por piso de tierra o pavimento. La vegetación se encuentra dispuesta en parches (de 2 a $15 \mathrm{~m}^{2}$ ) delimitados principalmente por concreto, la vegetación se compone principalmente de pasto y plantas ornamentales, así como árboles. La estructura de la vegetación está compuesta por hierba ( $\leq 0.5 \mathrm{~m}$ de altura), arbustos (1 a $1.5 \mathrm{~m}$ ) y árboles (3 a $15 \mathrm{~m}$ ). Entre las especies de árboles más comunes se encuentran Mangifera indica (mango) y Ficus benjamina (benjamina). En relación con el resto de las categorías su forma es simétrica, con una densidad de vegetación baja y su extensión es pequeña.

\section{Zanjones}

Estas son áreas ubicadas en una depresión de más de dos metros en contraste con el suelo circundante, rodeados de zonas habitacionales y son utilizados como sitios recreativos. Cuentan con abundante vegetación, la cual por lo general es nativa, pero en ocasiones se encuentran especies introducidas (ornamental o frutal). La estructura está compuesta por hierbas, arbustos y árboles. En relación con el resto de las categorías su forma es irregular, pero siempre alargada, con una densidad de vegetación alta y extensión mediana.

\section{Parques}

Son áreas que presentan un cuerpo de agua natural o artificial, este suele encontrarse al centro del parque rodeado de vegetación. La vegetación presente en estas áreas es abundante y en su mayoría es nativa. La altura de la vegetación suele ser de tres tipos: hierbas, arbustos y árboles; los arbustos son dominantes. Los parques son áreas de gran extensión que van desde 2 hasta 25 hectáreas y cuentan con muy pocas construcciones en ellos. Las zonas de los alrededores suelen ser zonas habitacionales y comerciales. En relación con el resto de las categorías su forma es simétrica, con una densidad de vegetación alta y su extensión es grande (Figura 2).

\section{Trabajo de campo}

Ante la dificultad de efectuar colectas de murciélagos en sitios públicos de la zona urbana, se optó por un diseño de muestreo sencillo, pero suficiente, para obtener información que permita cumplir con los objetivos de la investigación. El trabajo de campo consistió en realizar únicamente un muestreo (sin repeticiones) en cada 

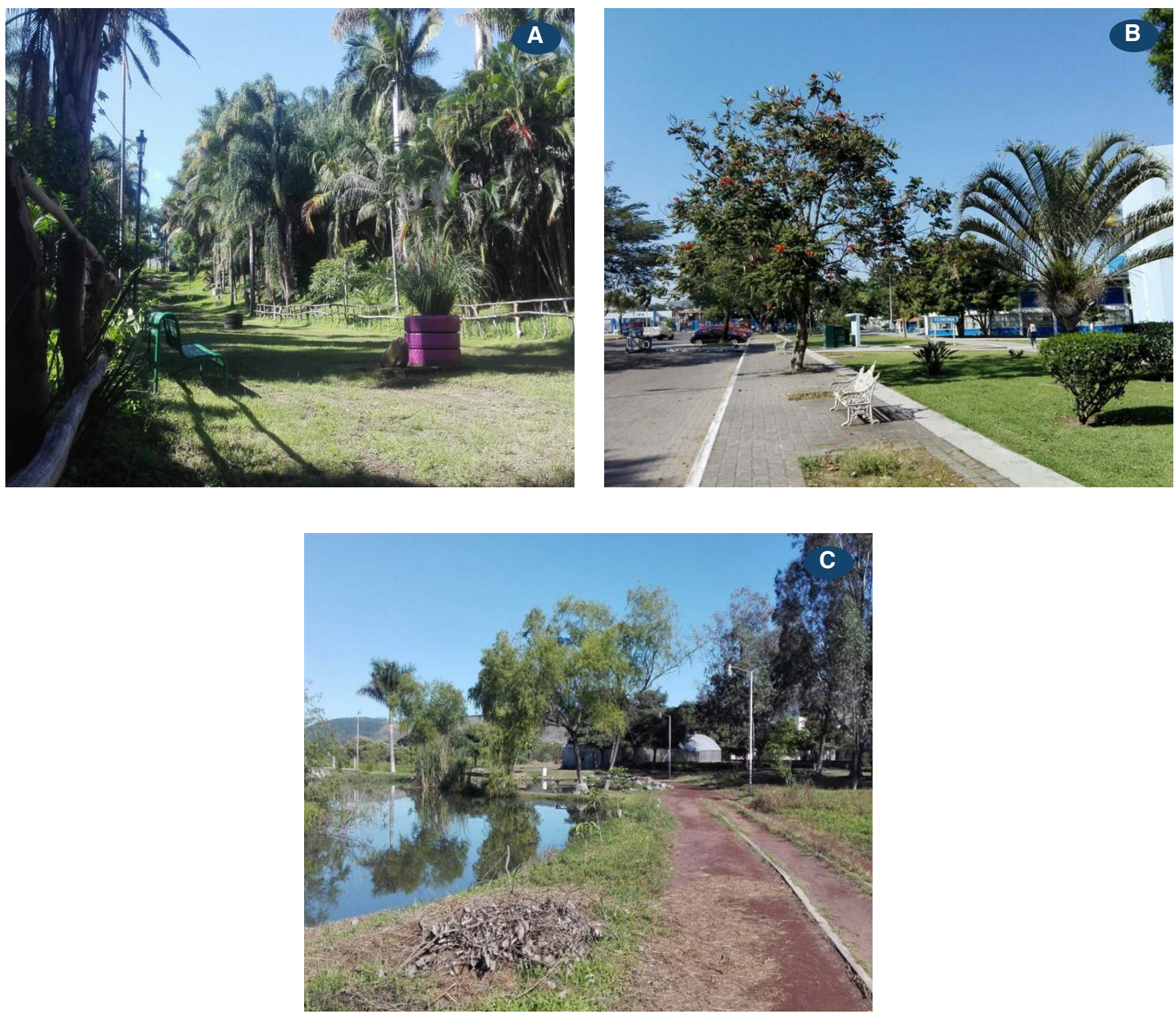

Figura 1. Ejemplo de los sitios de muestreo y ecosistema urbano al que corresponden: A) Zanjón Jaracarandas, B) plaza en la explanada de la Universidad Autónoma de Nayarit y C) parque ecológico de la ciudad de Tepic Nayarit. Fotos: Elsa Jazmín Lugo.

sitio de colecta y emplear el mismo esfuerzo de muestreo en cada uno de ellos, con el propósito de efectuar una comparación entre los sitios explorados.

Durante el periodo del 28 de noviembre del 2015 al 10 de noviembre del 2016 se efectuaron muestreos en nueve sitios de colecta de la zona urbana del municipio de Tepic, Nayarit, de los cuales tres corresponden a plazas (Calzada Panteón, Panteón de la Cruz y Explanada UAN), tres a zanjones (Zanjón Jacarandas, Zanjón Esperanza y Zanjón Lindavista) y tres a parques
(Parque Ecológico, Parque Metropolitano y Canal P. de Hamburgo; Cuadro 1). Los sitios de colecta fueron elegidos con base en observaciones previas, que fueron resultado en la selección de localidades con mayor avistamiento de murciélagos en las zonas verdes de la ciudad, solo se excluyeron dos parques debido a la dificultad de su acceso (la Loma y la Alameda).

Para el registro de especies se capturaron ejemplares por medio de una red de niebla de 12 metros de largo y 2.5 metros de alto, colocada a una altura de 3 metros, sujetada por postes 


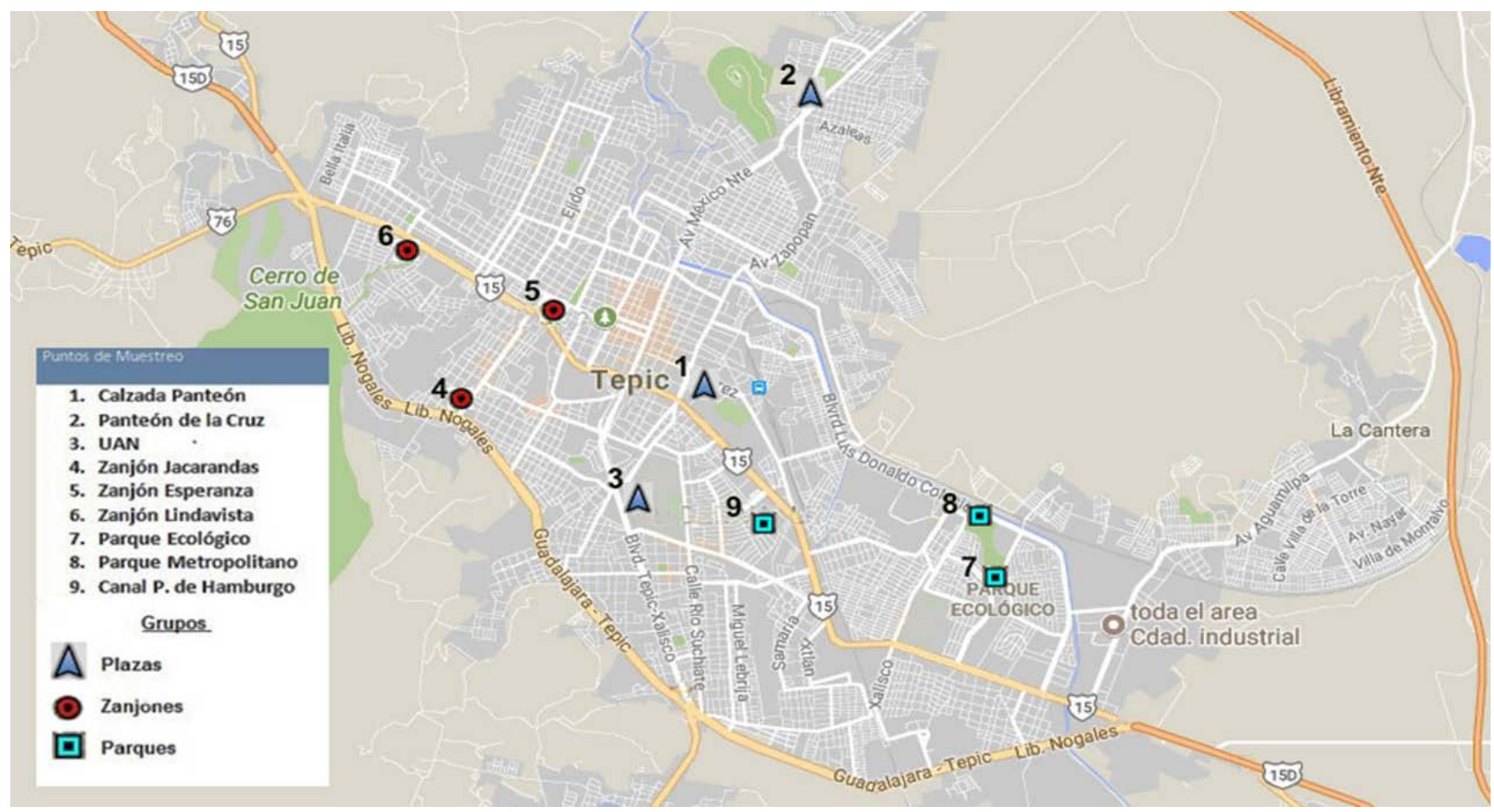

Figura 2. Ubicación de las áreas verdes de la zona urbana de Tepic, Nayarit, que fueron empleados como sitios de colecta de quirópteros. Se distinguen los distintos tipos de ecosistemas urbanos a los que pertenecen (plazas, zanjones y parques).

de aluminio. La red fue colocada de 18:00 $\mathrm{h}$ a 23:00 h, (se hizo un ajuste al término del horario de verano) y fue revisada cada 30 minutos para retirar los ejemplares capturados. El esfuerzo de muestreo acumulado fue de 45 horas/red y 108 metros/red. Los organismos capturados fueron identificados in situ, con base en sus características morfológicas externas y posteriormente fueron liberados. Solo algunos murciélagos fueron preparados para su incorporación al Museo de Zoología de la Universidad Autónoma de Nayarit (Cuadro 2). Para su identificación se emplearon las claves de Álvarez, et al., (1994), Álvarez-Castañeda et al. (2015), así como las descripciones de los artículos de Mammalian Species (Ortega y Castro, 2001; Webster y Jones, 1983).

En la determinación de especies se observaron algunas características diagnósticas importantes. Por ejemplo, los incisivos inferiores, el desarrollo de los procesos preorbital y postorbital, la longitud del cráneo y la fórmula dental, las cuales junto con las diferentes tonalidades del pelo dorsal y características del uropatagio ayudaron a elaborar una guía para la identificación en campo basado en características externas.

\section{Análisis de datos}

Se realizó un registro de las especies y número de individuos capturados, con lo cual se estimó la riqueza específica y la abundancia relativa (Abundancia relativa=No. de individuos/ No. total de individuos) de cada especie y cada sitio de colecta.

Posteriormente, se estimó para cada ecosistema urbano (plaza, zanjón y parque) la abundancia relativa (no. de individuos por especie/ no. total de individuos) y la frecuencia de murciélagos (no. de muestras en las que aparece la especie / no. total de muestreos). Así como la diversidad alfa, mediante la riqueza específica (S) y el índice de Shannon $(\mathrm{H})$, la fórmula utilizada fue la siguiente:

$$
\mathrm{H}=-\sum_{i} \frac{n_{i}}{n} \ln \frac{n_{i}}{n}
$$


Dónde: $\mathrm{n}=$ Número de individuos de la muestra y $n=e l$ número de individuos totales.

Por último, se realizó la prueba t modificada por Hutchenson, para comparar las diferencias que se presentan entre los ecosistemas urbanos mediante la siguiente fórmula:

H'= índice de Shannon de cada muestra

Var H'= varianza del índice de Shannon

$$
t=\frac{H_{1}^{\prime}-H_{2}^{\prime}}{\sqrt{\operatorname{Var} H_{1}^{\prime}}+\operatorname{Var} H_{2}^{\prime}}
$$

\section{Resultados}

\section{Diversidad de especies}

En total se capturaron 58 individuos en la zona urbana de Tepic, correspondientes a tres especies: el murciélago frugívoro gigante (Artibeus lituratus), el murciélago frutero (Artibeus jamaicensis) y el murciélago frugívoro peludo (Artibeus hirsutus; Cuadro 1).

Se observó que la especie más abundante fue el murciélago frugívoro gigante ( $A$. lituratus) con 45 individuos capturados, lo cual representa más del 70 por ciento de la abundancia en la zona, seguida del murciélago frutero $(A$. jamaicensis) con 10 individuos, que corresponde aproximadamente un 17 por ciento y solo tres capturas del murciélago frugívoro peludo, con un cinco por ciento (A. hirsutus; Figuras 3A, 3B y 3C).

Cuadro 1. Número de ejemplares capturados de cada especie de murciélago en las áreas verdes de la ciudad de Tepic, Nayarit. En el cuadro se indica la especie, los sitios de colecta y los ecosistemas urbanos que fueron estudiados.

\begin{tabular}{|c|c|c|c|c|c|c|c|}
\hline & Sitio & $\begin{array}{l}\text { Clave del } \\
\text { sitio }\end{array}$ & $\begin{array}{l}\text { Artibeus } \\
\text { lituratus }\end{array}$ & $\begin{array}{c}\text { Artibeus } \\
\text { jamaicensis }\end{array}$ & $\begin{array}{l}\text { Artibeus } \\
\text { hirsutus }\end{array}$ & $\begin{array}{l}\text { Individuos } \\
\text { por sitio }\end{array}$ & $\begin{array}{l}\text { Especies } \\
\text { por sitio }\end{array}$ \\
\hline \multirow{3}{*}{ Plaza } & $\begin{array}{l}\text { Calzada } \\
\text { Panteón }\end{array}$ & P1 & 1 & 2 & 0 & 3 & 2 \\
\hline & $\begin{array}{c}\text { Panteón de la } \\
\text { Cruz }\end{array}$ & P2 & 4 & 0 & 0 & 4 & 1 \\
\hline & $\begin{array}{l}\text { Explanada } \\
\text { UAN }\end{array}$ & P3 & 9 & 0 & 0 & 9 & 1 \\
\hline \multirow{3}{*}{ Zanjón } & $\begin{array}{c}\text { Zanjón } \\
\text { Jacarandas }\end{array}$ & Z1 & 4 & 0 & 0 & 4 & 1 \\
\hline & $\begin{array}{l}\text { Zanjón } \\
\text { Esperanza }\end{array}$ & Z2 & 6 & 0 & 0 & 6 & 1 \\
\hline & $\begin{array}{l}\text { Zanjón } \\
\text { Lindavista }\end{array}$ & Z3 & 9 & 2 & 0 & 11 & 2 \\
\hline \multirow{4}{*}{ Parque } & $\begin{array}{l}\text { Parque } \\
\text { Ecológico }\end{array}$ & Q1 & 3 & 1 & 1 & 5 & 3 \\
\hline & $\begin{array}{c}\text { Parque } \\
\text { Metropolitano }\end{array}$ & Q2 & 4 & 0 & 0 & 4 & 1 \\
\hline & $\begin{array}{l}\text { Canal P. de } \\
\text { Hamburgo }\end{array}$ & Q3 & 5 & 5 & 2 & 12 & 3 \\
\hline & $\begin{array}{l}\text { Individuos por } \\
\text { especie }\end{array}$ & & 45 & 10 & 3 & & \\
\hline
\end{tabular}



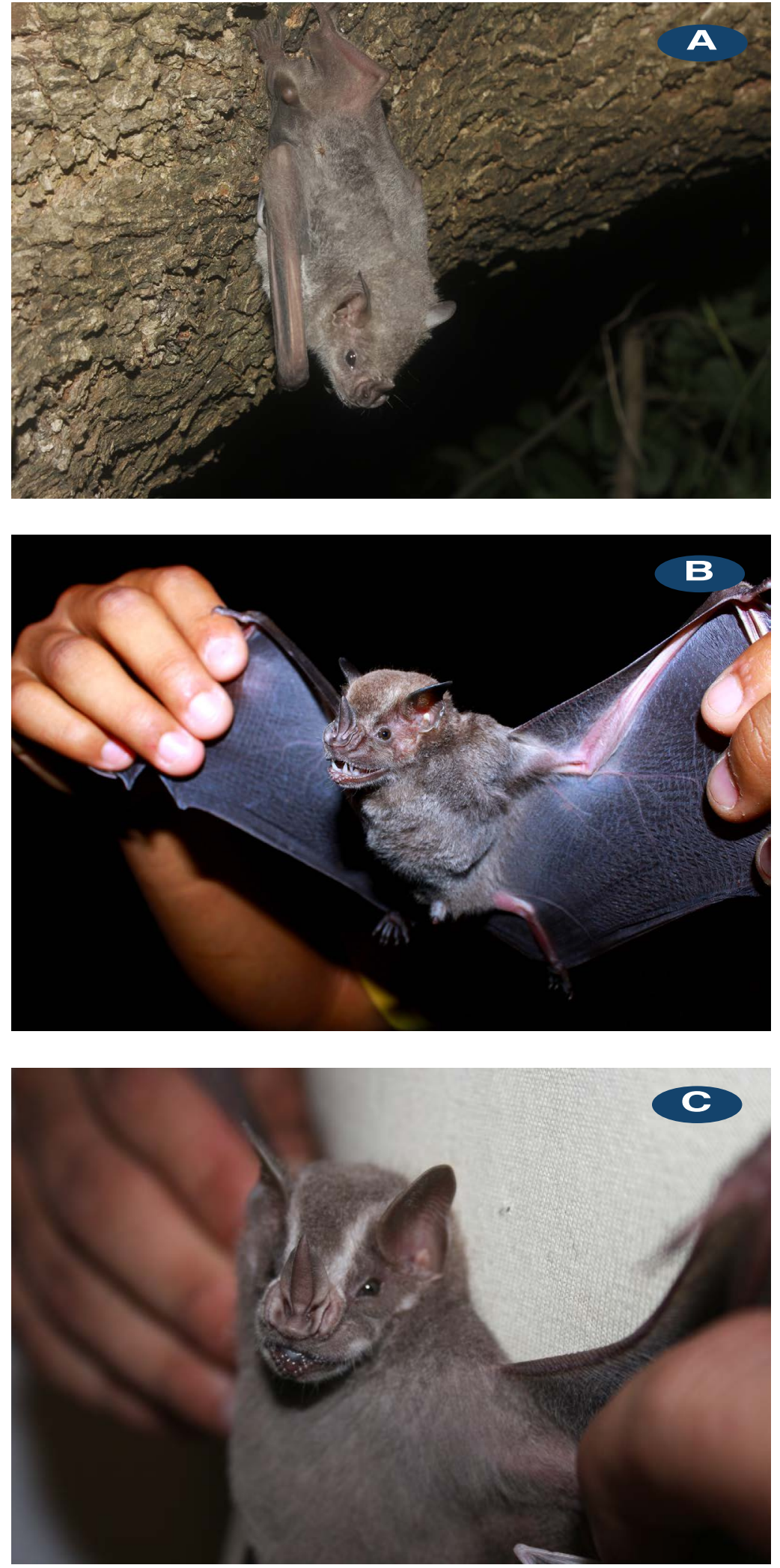

Figura 3. Especies de murciélagos encontrados en los tres sitios de muestreo. A) Artibeus hirsutus (Foto: Juan Cruzado https://www.inaturalist.org/photos/64849017), B) A. jamaicensis (Foto: Horacio Bárcenas https://www.inaturalist.org/photos/66825308) y C) A. lituratus (Foto: Horacio Bárcenas http:// conabio.inaturalist.org/photos/6518816). 
Cuadro 2. Ejemplares colectados de las tres especies del género Artibeus resguardados en el Museo de Zoología de la Universidad Autónoma de Nayarit.

\begin{tabular}{|c|c|c|c|c|}
\hline No. de catálogo & Sexo & Especie & Material & Localidad \\
\hline MZUAN-097 & Hembra & A. lituratus & Piel y cráneo & $\begin{array}{c}\text { Nayarit. Tepic. } \\
\text { Zanjón Lindavista }\end{array}$ \\
\hline MZUAN-098 & Macho & A. lituratus & Piel y cráneo & $\begin{array}{c}\text { Nayarit. Tepic. } \\
\text { Zanjón Lindavista }\end{array}$ \\
\hline MZUAN-099 & Hembra & A. lituratus & Piel y cráneo & $\begin{array}{c}\text { Nayarit. Tepic. } \\
\text { Zanjón Esperanza }\end{array}$ \\
\hline MZUAN-100 & Hembra & A. jamaicensis & Piel y cráneo & $\begin{array}{l}\text { Nayarit. Tepic. } \\
\text { Calzada del Panteón }\end{array}$ \\
\hline MZUAN-101 & Macho & A. jamaicensis & Piel y cráneo & $\begin{array}{c}\text { Nayarit. Tepic. } \\
\text { Calzada del Panteón }\end{array}$ \\
\hline MZUAN-102 & Macho & A. hirsutus & Piel y cráneo & $\begin{array}{c}\text { Nayarit. Tepic. } \\
\text { Canal P. de Hamburgo }\end{array}$ \\
\hline MZUAN-103 & Macho & A. hirsutus & Piel y cráneo & $\begin{array}{c}\text { Nayarit. Tepic. } \\
\text { Parque Ecológico }\end{array}$ \\
\hline
\end{tabular}

\section{Diversidad entre sitios de muestro}

Los sitios con mayor número de especies fueron Q1 y Q3, ambos con tres especies; en contraste, los sitios con menor número de especies son el P2, P3, Z1, Z2 y Q2 con una sola especie. Sin embargo, los sitios Q3 y Z3 presentaron la mayor abundancia relativa de murciélagos, mientras que P1, P2, Z1 y Q1 la menor abundancia. De tal manera que el Canal de Hamburgo (Q3) es el sitio con mayor número de especies y de organismos observados en este estudio, mientras que el Panteón de la Cruz (P2) y la explanada de la UAN (P3) son los lugares más pobres en cuanto a diversidad de murciélagos (Cuadro 1).

\section{Comparacion entre ecosistemas urbanos}

El tipo de ecosistema urbano que presentó mayor riqueza especifica son los parques, en los cuales se documentó la presencia de tres especies ( $A$. hirsutus, $A$. lituratus y $A$. jamaicensis), mientras que en las plazas y zanjones se observó la presencia de dos especies en cada uno de ellos (A. lituratus y A. jamaicensis).

La especie que presentó el valor de frecuencia más elevado fue $A$. lituratus (con un valor de 1.0), por lo que es la especie más común, ya que se registró en todos los sitios de colecta de todos ecosistemas analizados, seguida por $A$. ja- maicensis (con un valor de 0.44), que se observó en los tres tipos de ecosistemas urbanos, pero con un menor número de capturas. La especie menos frecuente fue $A$. hirsutus (con un valor de 0.22 ), pues se registraron muy pocas capturas y solo se presentó en parques (Cuadro 1).

La mayor diversidad alfa de acuerdo con estimador de Shannon se obtuvo en el ecosistema urbano denominado parques (0.9557), seguido por plazas $(0.3768)$ y el valor más bajo se observó en zanjones (0.3145). La prueba de t modificada por Hutchenson, indicó que plazas y zanjones no muestran diferencias significativas ( $\mathrm{t}=$ 24581 y df= 34.721, $P=0.8072$ ). Sin embargo, la comparación entre plazas y parques $(\mathrm{t}=-2.737 \mathrm{y}$ $\mathrm{df}=30.602, \mathrm{P}=0.0102)$, así como entre zanjones y parques $(t=-3.2388$ y df $=40.274, P=0.0024) \mathrm{si}$ muestra diferencias significativas; por lo que se determinó que parques, es el tipo de ecosistema que presenta mayor diferencia con respecto a los otros ecosistemas analizados.

\section{Discusión}

La cuidad de Tepic presenta una diversidad de murciélagos similar a la reportada por otros autores en lugares con características similares, tal es el caso de García-Méndez et al. (2014), quienes reportan tres especies de murciélagos en áreas verdes de San Cristóbal de las Casas, 
Chiapas, y Silvio (2019) que ha documentado recientemente para la Ciudad de México la presencia de cuatro especies de murciélagos. Es probable que esta baja diversidad sea el resultado de la sensibilidad que estos organismos presentan ante la deforestación y fragmentación de los espacios verdes, que disminuye y reduce sus poblaciones (Viveros, 2010).

Es importante señalar que durante el trabajo de campo se observaron murciélagos insectívoros, los cuales no fueron capturados con la metodología empleada, puesto que la mayoría de las especies de murciélagos insectívoros vuelan sobre el estrato arbóreo o bien a alturas superiores a la que generalmente se colocan las redes (Ortegón-Martínez y Pérez-Torres, 2007).

La abundancia de murciélagos en las zonas urbanas en gran medida está determinada por la presencia de zonas de descanso y alimentación (Padilla y Cuesta, 2003). Sin embargo, no todas las especies tienen los mismos requerimientos de hábitat, algunas demandan condiciones más específicas, lo cual restringe su presencia (Fenton et al., 1992), mientras que otras son más generalistas y se observan con mayor frecuencia en ambientes modificados. Tal es el caso del género Artibeus que suele presentarse comúnmente en zonas urbanas ya que, gracias a su gran masa corporal, que les permite tener una mayor fuerza de mordida, son capaces de consumir frutos de menor calidad, duros y con poco jugo (Saldaña-Vázquez y Schondude, 2016).

Artibeus lituratus, la especie más común registrada en Tepic es flexible en cuanto al uso de refugios en comparación con otros filostómidos, ya que puede ocupar refugios de baja calidad, con alta humedad, cambios de temperatura, y de mayor luminosidad (Ortiz-Ramírez et al., 2006). Por lo tanto, esta especie suele ocupar refugios que no son óptimos para otras especies, lo que genera una mayor abundancia y frecuencia de individuos en la localidad. Por su parte Artibeus jamaicensis, es la especies más ampliamente distribuida y abundante del género Artibeus, suele encontrarse en ambientes tan diversos como los bosques húmedos tropicales, secos tropicales, bosques nublados, secos y ambientes modificados por el humano. Esta especie es frugívora generalista, consume alrededor de 127 especies de plantas frutales, entre las cuales se encuentran Psidium guajava, Mangifera indica y F. ben- jamina, aunque suelen tener preferencia por los Ficus (Ortega y Castro, 2001).

En cuanto al uso de ecosistemas urbanos, se observó que los zanjones presentan la menor diversidad de murciélagos. A pesar de que ostentan una gran diversidad de vegetación, su forma no es la óptima ya que son lugares con una gran longitud, pero el ancho es muy reducido, lo que incrementa el efecto de borde y se ha documentado que la forma y la superficie del hábitat es un factor importante para la presencia de murciélagos (Sierra, 2012). En contraste, los parques, son el ecosistema que mostró la mayor diversidad de murciélagos; este ecosistema se caracteriza por contar con un área de gran tamaño, en la cual existe una gran diversidad de vegetación, que ofrece alternativas de alimentación y refugio. Asimismo, son el único ecosistema en el que se registró la especie $A$. hirustus. Probablemente esto está asociado a la presencia de cuerpos de agua (presentes únicamente en Parques), pues esta especie en condiciones naturales es capturada regularmente cerca de cuerpos de agua (Webster y Jones, 1983).

La transformación del paisaje y los procesos de urbanización disminuyen el hábitat de los murciélagos, las áreas verdes se convierten en zonas prioritarias en donde estas especies pueden realizar sus actividades de descanso y alimentación (Garcés-Restrepo et al., 2016). Aunque la principal función de los parques, plazas y zanjones está relacionada con la recreación de la población humana, es importante considerar que estos sitios cumplen una función importante en la conservación de la flora y la fauna silvestre.

\section{Conclusiones}

La zona urbana de Tepic cuenta con tres especies de murciélagos ( $A$. hirsutus, $A$. lituratus y $A$. jamaicensis). Particularmente los parques son los ecosistemas urbanos que presentaron una mayor diversidad de murciélagos y que adicionalmente presenta diferencias significativas con respecto a los zanjones y plazas. El murciélago frutero gigante ( $A$. lituratus) es la especie más abundante y frecuente en la zona urbana; probablemente por su tamaño, sus hábitos de alimentación y tolerancia a condiciones adversas, que le permiten prevalecer en zonas altamente perturbadas. 


\section{Agradecimientos}

A la Dirección de Ecología y Protección al Medio Ambiente del municipio de Tepic, y la Dirección de Parques y Jardines del Municipio de Tepic, por las facilidades otorgadas, así como a los estudiantes del Museo de Zoología de la UAN por su apoyo en el trabajo de campo. Las colectas fueron realizadas bajo el permiso SGO/ DGVS/06719/16 a nombre de Juan Pablo Ramírez Silva.

\section{LITERATURA CITADA}

Álvarez, T., T. Álvarez-Castañeda y J.C. López-Vidal. 1994. Claves para Murciélagos Mexicanos. Centro de Investigaciones Biológicas del Noroeste, S.C. y Escuela de Ciencias Biológicas I.P.N., México, D.F.

Álvarez-Castañeda, T., T. Álvarez y N. González-Ruiz. 2015. Guía para identificar los mamíferos de México. Talleres de Pandora Impresores, S.A. de C.V. México.

Arias-Aguilar, A., E. Chacón-Madrigal y B. Rodríguez-Herrera. 2015. El uso de los parques urbanos con vegetación por murciélagos insectívoros en San José, Costa Rica. Mastozoología Neotropical, 22:229-237.

Díaz, M., M. Carbajal, E. Lipps, M. Lutz, S. Rosenfeld y R. Barquez. 2013. El estado de conservación de los murciélagos en Argentina, Pp. 273-281, en: Manejo de Fauna Silvestre en la Argentina. Programas de Conservación de especies amenazadas (Porini, G. y D. Ramadori, eds.). Dirección de Fauna Silvestre, Secretaría de Ambiente y Desarrollo Sustentable. Buenos Aires, Argentina.

Fenton, M., L. Acharya, D. Audet, M. Hickey, C. Merriman, M. Obrist y B. Adkins.1992. Phyllostomid Bats (Chiroptera: Phyllostomidae) as Indicators of Habitat Disruption in the Neotropics. Biotropica, 24:440-446. [doi:10.2307/2388615]

Garcés-Restrepo, M., A. Giraldo., C. López y N. Ospina-Reina. 2016. Diversidad de Murciélagos del Campus Meléndez de La Universidad del Valle, Santiago de Cali, Colombia. Boletín Científico Museo de Historia Natural, 20:116-125.
García-Méndez, A., C. Lorenzo., L.B. Vázquez, y R. Reyna-Hurtado. 2014. Roedores y murciélagos en espacios verdes en San Cristóbal de Las Casas, Chiapas, México. Therya, 5:615-632. [DOI:10.12933/therya-14-207]

Gonsalves, L., S. Lamb, C. Webb, B. Law y B. Monamy. 2013. Do mosquitoes influence bat activity in coastal habitats? Wildlife Research, 40:10-24.

Greenhall, A.M. 1982. House bat management. United States Departmen of the Interior, Fish and Wildlife Service. Washington D.C., Unites States of America.

Hourigan, C.H., C. Johnson y S. Robson. 2006. The structure of a micro-bat community in relation to gradients of environmental variation in a tropical urban area. Urban Ecosystem, 9:67-82.

INEGI. 2010. México en Cifras [internet] Aguascalientes, México. INEGI. Disponible en:<https:// www.inegi.org. $\mathrm{mx} / \mathrm{app} /$ areasgeograficas/?ag=18> [Consultado el 30 de agosto de 2018].

INEGI. 2017. Conociendo Nayarit. 7a. ed. Instituto Nacional de Estadística y Geografía. México.

Jung, K. y C.G. Threlfall. 2016. Urbanisation and Its Effects on Bats-a global meta-analysis. Pp. 13-33, en: Bats in the Anthropocene: conservation of bats in a changing World. (Voigt, C. y T. Kingston, eds.) Springer, Cham.

Luck, G., L. Smallbone, C. Threlfall, y B. Law. 2013. Patterns in bat functional guilds across multiple urban centres in south-eastern Australia. Landscape Ecology, 28:455-469.

Ortega, J. y I. Castro. 2001. Artibeus jamaicensis. Mammalian Species, 662:1-9.

Ortegón-Martínez, D.A. y J. Pérez-Torres. 2007. Estructura y composición del ensamblaje de murciélagos (Chiroptera) asociado a un cafetal con sombra en la Mesa de los Santos (Santander), Colombia. Actual Biology, 29:221-234.

Ortiz-Ramírez, D., C. Lorenzo, E. Naranjo y L. León-Paniagua. 2006. Selección de refugios por tres especies de murciélagos frugívoros (Chiroptera Phyllostomidae) en la Selva La- 
candona, Chiapas, México. Revista Mexicana de Biodiversidad, 77:261-270.

Padilla, F. y A. Cuesta. 2003. Zoología Aplicada. España: Ediciones Díaz de Santos.

Rhodes, M. y C. Catterall. 2008. Spatial foraging behaviour and use of an urban landscape by a fast-flying bat, the Molossid Tadarida australis. Journal of mammalogy, 89:34-42.

Saldaña-Vázquez, R.A. y J.E. Schondube. 2016. La masa corporal explica la dominancia de Artibeus (Phyllostomidae) en ambientes urbanos. Pp. 23-33, en: Fauna Nativa en Ambientes Antropizados (Ramírez-Bautista A. y R. Pineda-López, eds.). CONACYT-Universidad Autónoma de Querétaro. Querétaro, México.

Sierra, M.A. 2012. Ciudad y fauna urbana. Un estudio de caso orientado al reconocimiento de la relación hombre, fauna y hábitat urbano en Medellín. Tesis de Maestría. Medellín, Universidad Nacional de Colombia. Colombia.

Silvio, R. 2019. Local: Las 4 especies de murciélago en la ciudad y dónde visitarlas [internet], México, D.F., Ortiz, L. Disponible en: $<$ https://local.mx/ciudad-de-mexico/medio-ambiente/murcielagos-ciudad/>. [Consultado el $11 \mathrm{de}$ febrero 2020].

Threlfall, C., G. Law, y P. Banks. 2012. Influence of landscape structure and human modifications on insect biomass and bat foraging activity in an urban landscape. PloS One, 7:1-10.

Viveros, J.C. 2010. Diversidad alfa y abundancia de los murciélagos de hoja nasal (Phyllostomidae) en Xpujil, Campeche. Tesis profesional de licenciatura, Universidad Veracruzana. México.

Webster, D. y K. Jones. 1983. Artibeus hirsutus and Artibeus inopinatus. Mammalian Species, 199:1-3. 